\title{
Current Applications of Genetic Risk Scores to Cardiovascular Outcomes and Subclinical Phenotypes
}

\author{
Jennifer A. Smith ${ }^{1} \cdot$ Erin B. Ware $^{1,2} \cdot$ Pooja Middha $^{1} \cdot$ Lisa Beacher $^{1}$. \\ Sharon L. R. Kardia ${ }^{1}$
}

Published online: 1 July 2015

(C) The Author(s) 2015. This article is published with open access at Springerlink.com

\begin{abstract}
Genetic risk scores are a useful tool for examining the cumulative predictive ability of genetic variation on cardiovascular disease. Important considerations for creating genetic risk scores include the choice of genetic variants, weighting, and comparability across ethnicities. Genetic risk scores that use information from genome-wide meta-analyses can successfully predict cardiovascular outcomes and subclinical phenotypes, yet there is limited clinical utility of these scores beyond traditional cardiovascular risk factors in many populations. Novel uses of genetic risk scores include evaluating the genetic contribution of specific intermediate traits or risk factors to cardiovascular disease, risk prediction in highrisk populations, gene-by-environment interaction studies, and Mendelian randomization studies. Though questions remain about the ultimate clinical utility of the genetic risk score, further investigation in high-risk populations and new
\end{abstract}

This article is part of the Topical Collection on Cardiovascular Disease

Jennifer A. Smith

smjenn@umich.edu

Erin B. Ware

ebakshis@umich.edu

Pooja Middha

pmiddha@umich.edu

Lisa Beacher

lbeacher@umich.edu

Sharon L. R. Kardia

skardia@umich.edu

1 Department of Epidemiology, School of Public Health, University of Michigan, 1415 Washington Heights, Ann Arbor, MI 48109, USA

2 Research Center for Group Dynamics, Institute for Social Research, University of Michigan, Ann Arbor, MI 48104, USA ways to combine genetic risk scores with traditional risk factors may prove to be fruitful.

Keywords Genetic risk score - Cardiovascular disease . Coronary heart disease $\cdot$ Ischemic stroke $\cdot$ Hypertension . Blood pressure

\section{Introduction}

Multi-cohort genome-wide association studies (GWAS) have now identified hundreds of genetic variants that are credibly associated with cardiovascular outcomes, subclinical cardiovascular phenotypes, and risk factors for cardiovascular disease (CVD). However, the individual genetic variants, or single nucleotide polymorphisms (SNPs), that have been identified typically explain a very small fraction of the variation in complex traits and thus have limited predictive capacity for disease risk [1]. Aggregating information about multiple SNPs, each with small effects, into a single genetic risk score (GRS) has become a useful tool for examining the cumulative predictive ability of genetic variation at known loci on cardiovascular disease outcomes and related phenotypes [2].

Here, we outline key aspects of creating GRSs, discuss their quantification and evaluation, and provide a brief summary of the predictive ability of GRSs for cardiovascular outcomes and subclinical CVD phenotypes. Emerging uses of GRSs will then be discussed, including (1) prediction in clinical and high-risk populations, (2) GRS-by-environment interaction studies, and (3) Mendelian randomization studies. Cardiovascular outcomes discussed include coronary heart/artery disease (CHD/CAD), myocardial infarction (MI), ischemic stroke (IS), hypertension (HTN), and a composite CVD phenotype that includes both heart disease and stroke (also conceptualized as "total cardiovascular diseases" [3]). Subclinical 
phenotypes include artery calcification and intimal-medial thickness (IMT).

\section{Creating Genetic Risk Scores}

Fundamentally, the creation of a GRS involves summarizing information across multiple SNPs. The most common method sums the number of risk-conferring alleles that an individual has $(0,1$, or 2$)$ across all loci. A statistically analogous coding scheme is to assign the heterozygous state (i.e., Aa) a value of 0 , the non-risk homozygous state -1 , and the risk homozygous state 1 . If an individual is missing a small proportion of genotype data needed to construct the GRS (such as 1 or 2 SNPs), imputation to the most common genotype category is commonly used. An alternative to imputation is to only include SNPs that have complete genotype data in the GRS, followed by GRS rescaling to be consistent with GRSs created using all SNPs.

\section{SNP Selection}

To create a GRS, one must first select the genetic variants to be included in the risk score. Although earlier studies creating GRSs for cardiovascular traits included SNPs from biologically plausible candidate gene association studies [4, 5], most current GRSs are constructed using SNPs found to be associated with traits through GWAS. The standard in the field has been to use SNPs that reached genome-wide significance $\left(p<5 \times 10^{-8}\right)$ in large, consortium-based, multi-study GWAS meta-analyses. Typically, these consortium-based meta-analyses also include a replication phase, which further enhances the robustness of the findings. While selecting SNPs from large meta-analyses is considered the gold standard, less preferable strategies for SNP selection may be used if metaanalyses have not yet been conducted for the trait of interest in populations that are demographically and/or ethnically similar to the population under study (for example, SNPs may be selected from biologically plausible candidate genes or from a single-study GWAS). The vast majority of GRSs in the literature include only common variants (SNPs with minor allele frequency (MAF) $>5 \%$ ), because meta-analyses tend not to have sufficient power to detect effects from rarer variants.

A disadvantage of including only the most highly significant and replicated SNPs is that there may be many other SNPs with true effects that do not reach the stringent genome-wide significance levels. Recent work evaluating the relationship between coronary artery calcification (CAC) and GRSs constructed from CHD/MI-associated SNPs at various meta-analysis $p$ value thresholds showed that trait variation for CAC is maximally explained by including thousands of SNPs that are at least marginally associated with CAD/MI $(p<0.2)$ in the GRS [6•]. On the other hand, research on type 2 diabetes suggests that GRSs constructed from increasing numbers of SNPs may not substantively improve risk prediction [7]. With these limited, sometimes conflicting results, more work is needed to verify whether inclusion of additional marginally significant SNPs in GRSs is the best approach for cardiovascular traits.

It has also been suggested that relying solely on genomewide significant SNPs from the largest, most current metaanalysis may not be the best approach to SNP selection. New algorithms that integrate information from multiple sources into GRS SNP selection are beginning to appear. For example, Belsky and colleagues implemented a novel SNP selection method using public-access resources, including GWAS results databases and web-based GWAS analysis tools, to select SNPs from 16 published GWAS for an obesity GRS [8・•]. This type of algorithm may represent a systematic and replicable method for integrating results from a wider variety of sources.

\section{Weights}

When equal weights are assigned to each genetic variant, the score is "unweighted," and its construction is based on the assumption that each risk allele confers identical risk. However, for most complex traits, effect sizes across identified SNPs vary (see, for example, [9]). Thus, GRSs are often constructed by weighting SNPs by their GWAS meta-analysis effect sizes, thus giving more weight to variants with stronger effects. Weighted scores may increase statistical power compared to unweighted scores, provided that the weights are accurately determined [10॰•]. Weights are ideally calculated from consortium-based meta-analysis effect sizes, which are more precise due to large sample sizes. This weighting method is commonly used when the target population (the population in which the GRS is going to be evaluated) has a similar demographic and ethnic composition as the meta-analysis population (the population used to estimate the effect sizes). See below for a more detailed discussion of the importance of ethnicity in GRS creation. An unweighted score is often the best option if there are no stable effect estimates available because (1) no GWAS meta-analyses have yet been performed on the trait of interest (and thus SNPs are selected from candidate gene studies or small, un-replicated GWAS), (2) existing meta-analyses are comprised of studies with different ethnicities or demographic profiles than the population to be studied, or (3) SNPs identified using multiple traits on different measurement scales are to be combined into a single GRS (for example, a GRS that comprises SNPs associated with multiple "intermediate traits," described in greater detail below). 


\section{Population-Specific Considerations}

A potential disadvantage of selecting SNPs based on published meta-analysis results is that many meta-analyses for complex traits have been conducted solely in Europeanancestry (EA) populations. This practice is problematic because the SNPs most significantly associated with a trait often differ across ethnicities for a variety of reasons including (1) ethnicity-specific genetic variation, (2) allele frequency differences across ethnicities, and (3) differing patterns of linkage disequilibrium (LD) resulting in ethnicity-specific "tag SNPs" that are associated with the causal variant(s) [11]. Trans-ethnic meta-analyses for cardiovascular traits are beginning to emerge $[12,13]$ and may offer several advantages over single-ethnicity analyses [11]. However, until trans-ethnic analyses become commonplace, other approaches for GRS SNP selection may be required for GRSs constructed for use in non-EA populations. One strategy is to evaluate SNPs from EA meta-analyses for association within the target population and retain only those that have at least a marginal effect. SNPs selected in this manner may also be combined with the most highly significant SNPs from smaller ethnicity-specific GWAS meta-analyses (see, for example, [14, 15]).

Population-specific factors such as age, sex, and demographics may also be important considerations for SNP selection and weighting. While there is an awareness that confounding and effect modification by population-specific factors may influence both estimates and inferences, the field of genetics has been primarily concerned with race/ethnicity because of the way in which it fundamentally changes the variants that are included and identified in an analysis. Aside from ethnicity, GWASs often include populations with a large range of demographics in order to achieve the sample sizes necessary to obtain enough power to accurately identify SNPs. Nevertheless, glaring demographic differences should be considered when creating GRSs.

\section{Estimating and Evaluating Genetic Risk Score Effects}

The effect sizes for the associations between GRSs and cardiovascular phenotypes are typically reported as beta estimates, odds ratios (ORs), or hazard ratios (HRs), as appropriate for the type of outcome. Effects may be reported per risk allele (corresponding to a one-allele increase in GRS), per GRS standard deviation (SD), or with respect to a particular comparison such as the contrast between the highest and lowest GRS quartiles. Differing methods of reporting effects often makes comparison across GRS studies difficult. In addition, per allele effect sizes tend to decrease as newly discovered variants are incorporated into GRSs. This is due to smaller effect sizes of the newly discovered variants compared to those discovered in the first wave of GWAS meta-analysis, as has been demonstrated for type 2 diabetes [9]. Thus, reporting effects per GRS SD is more effective for crossstudy and cross-trait comparisons.

The contribution of GRSs to quantitative cardiovascular traits, such as CAC, is typically reported as the percent of variation in the trait explained by the GRS. This may be assessed before and after adjustment for traditional cardiovascular risk factors (e.g., body mass index (BMI), lipids, HTN, diabetes, and others). For clinical outcomes, such as CAD or HTN, the predictive capacity of the GRS is most commonly evaluated using metrics for risk discrimination and risk reclassification (reviewed in [16]). Prediction models are constructed before and after including traditional cardiovascular risk factors and/or family history of disease. If the GRS significantly improves prediction after inclusion of traditional risk factors, it demonstrates the potential for clinical utility through more accurate disease risk prediction for patient populations. The area under the receiver operating curve (AUC, or cstatistic) is commonly used to assess discrimination between people with and without disease [17-19]. The c-index is the analogous measure for survival data. Higher AUCs indicate more accurate discrimination, and model improvement is assessed by change in AUC across models. However, many have argued that AUC-based methods may not be optimal for predicting risk [19]. The net reclassification improvement index (NRI) is a popular choice for evaluating risk reclassification $[16,20,21,22 \bullet \bullet$. This statistic evaluates a prediction model's ability to correctly reassign individuals into disease classifications when compared to a different model. Positive values of NRI correspond to prediction improvement. Clinical NRI corresponds to correct risk reclassification of individuals at intermediate risk for disease and may be more clinically relevant than the traditional NRI [23]. Other methods for assessing the potential for clinical utility, such as the integrated discrimination improvement (IDI) [20], are also available.

\section{Applying GRSs to Cardiovascular Traits}

A variety of approaches have been used to examine the relationship between GRSs and cardiovascular traits. Below, we discuss the current literature on (1) CAD/CHD-associated SNPs predicting CAD/CHD, (2) blood pressure (BP)-associated SNPs predicting BP and HTN, both within and across ethnic groups, (3) intermediate trait-GRSs predicting cardiovascular outcomes, (4) GRSs predicting composite CVD, and (5) GRSs associated with subclinical measures of heart disease. Representative examples of studies that fall into each of these categories are provided for dichotomous cardiovascular outcomes and quantitative cardiovascular traits in Tables 1 and 2 , respectively. 


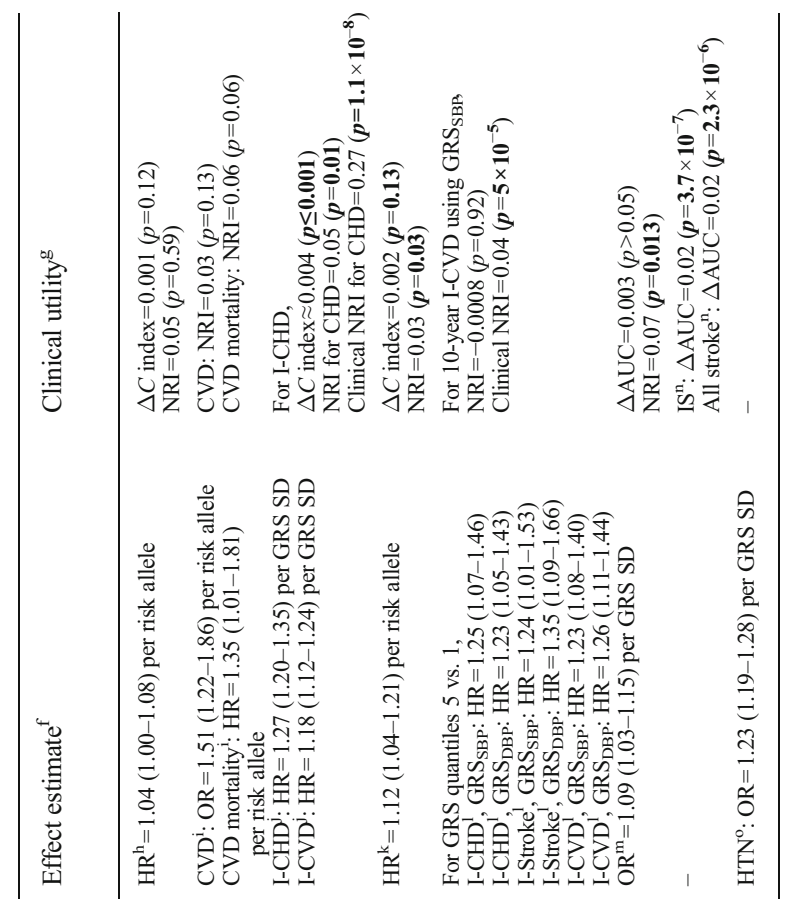

눙

응

总兽畗

产 旅

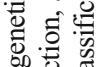

苍苟

量爱离

ํํㄹำ

政

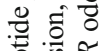

0
0
0
0

的总

का

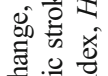

등 을

$\checkmark$ 过

은

尊

공

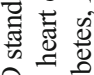

की

矛额

产令

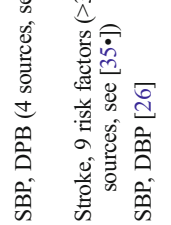

S)

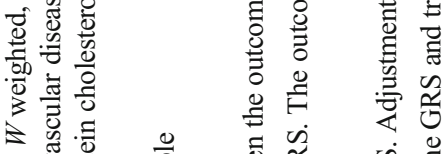

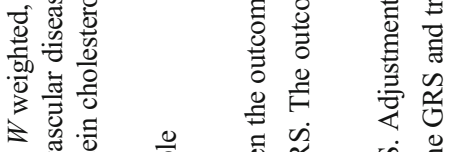

है

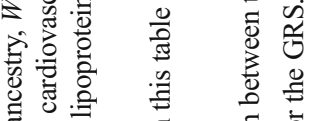

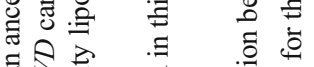

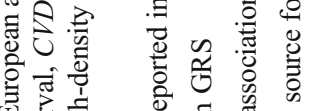

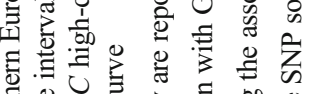

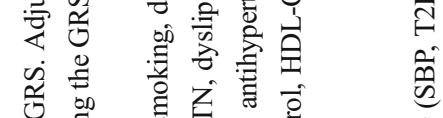

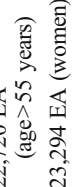

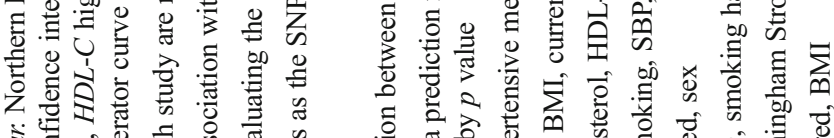

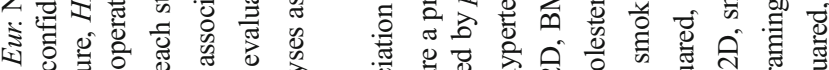

之心

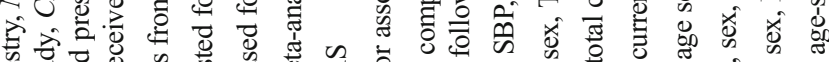

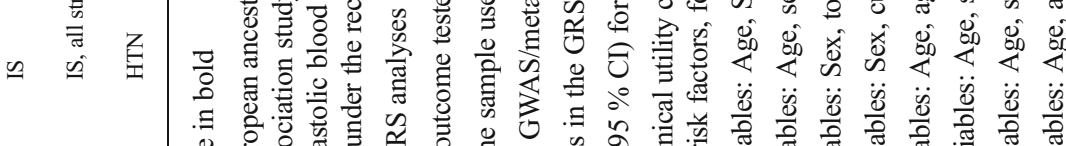

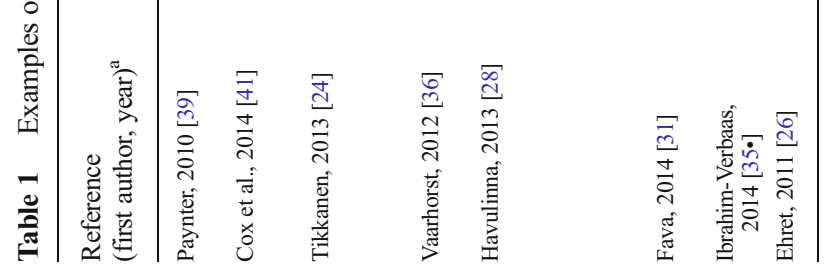

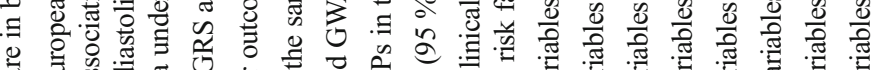

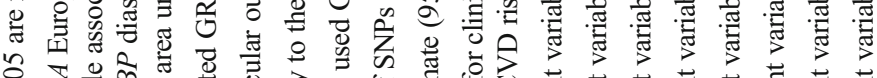

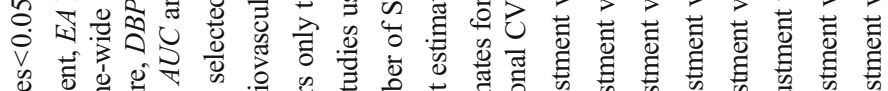

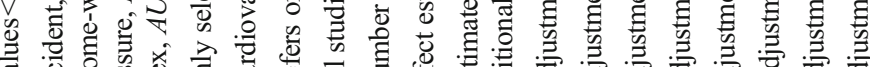

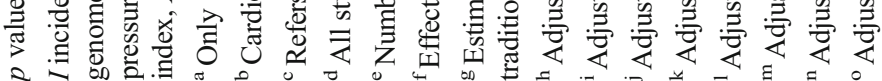




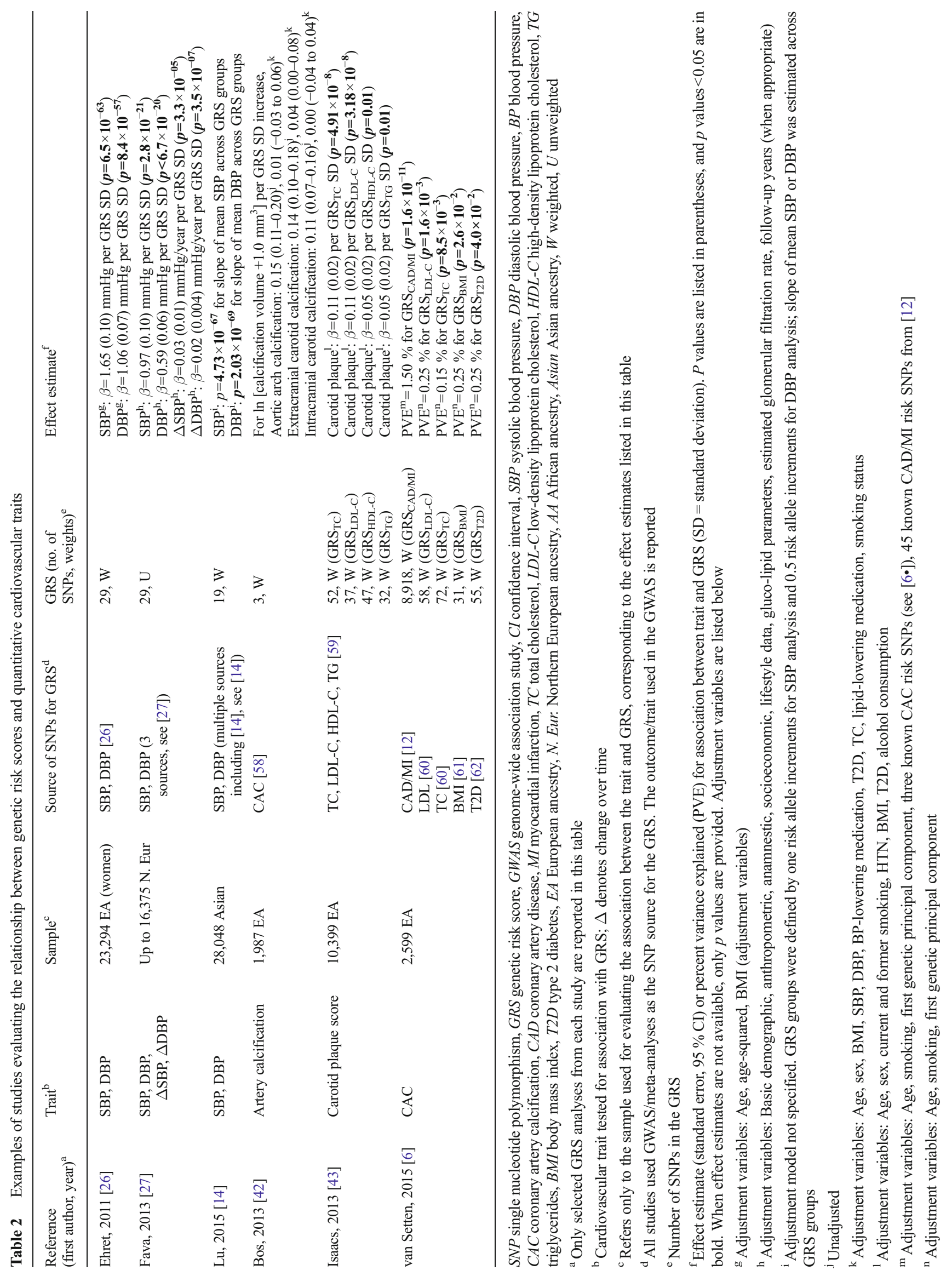




\section{CAD/CHD-Associated SNPs Predicting CAD/CHD}

The original concept of using GRSs to predict cardiovascular disease focused on using SNPs associated with the trait of interest to predict that same trait (i.e., CAD-GRS to predict CAD). Several studies have used SNPs found to be associated with CAD in consortium-based meta-analyses to construct GRSs for evaluation with respect to CAD/CHD in EA populations. For example, a weighted 24-SNP GRS constructed using CAD-associated SNPs from four meta-analyses was significantly associated with incident CHD in a Finnish cohort of $>24,000$ individuals ( $\mathrm{HR}=1.27$ per GRS SD) [24]. A study of $>10,000$ Swedes showed similar results for a 46-SNP GRS constructed using CAD-associated SNPs from the largest CAD meta-analysis to date [12] $(\mathrm{HR}=1.54$ for incident CHD comparing first versus fourth GRS quartile) [25]. In both studies, as is often the case when using GRSs to predict cardiovascular disease outcomes, models including the GRS modestly but significantly improved risk reclassification beyond traditional risk factors, but discrimination was not improved.

\section{BP-Associated SNPs Predicting BP and HTN}

The strength of association between GRSs constructed from BP-associated SNPs and HTN or BP has been examined in EA populations. In 2009, the International Consortium for Blood Pressure Genome-Wide Association Studies consortium (ICBP) conducted a GWAS meta-analysis of HTN and BP phenotypes in $>200,000$ EA [26]. A GRS was created from 29 SNPs associated with systolic blood pressure (SBP) and/or diastolic blood pressure (DBP) at $p<5 \times 10^{-9}$, weighted by the mean effect size for SBP and DBP. The GRS was evaluated in an independent cohort of 23,294 women and showed an increase of 1.65 and $1.10 \mathrm{mmHg}$ per SD of the GRS for SBP and DBP, respectively, as well as a $23 \%$ increase in the odds of HTN. When this same GRS was evaluated in a longitudinal study of $>17,000$ Swedes, a 1 SD increase in GRS was significantly associated with an increase of 1.0 and $0.6 \mathrm{mmHg}$ in SBP and DBP, respectively, as well as a $61 \%$ increase in the odds of hypertension at baseline [27]. The proportion of variation explained by the GRS was 1.0, 0.7, and $2.9 \%$ for SBP, DBP, and HTN, beyond traditional risk factors. Changes in SBP (beta $=0.03 \mathrm{mmHg}$ ), DBP (beta $=$ $0.023 \mathrm{mmHg})$, and HTN incidence $(\mathrm{OR}=1.11)$ were also significantly associated with the GRS. In this study, discrimination for HTN was marginally but not significantly improved by adding the GRS to traditional risk factors. These studies show that while GRSs created for BP and HTN are strongly associated with these traits, clinical utility may be limited.

GRSs consisting of BP-associated SNPs have also been evaluated in non-EA ethnicities. A trans-ethnic GWAS metaanalysis with an African-ancestry discovery sample and a multi-ethnic replication sample identified five SNPs credibly associated with blood pressure that were not previously identified through EA meta-analyses [13]. In the African-ancestry discovery sample, a weighted GRS with these five SNPs explained 0.44 and $0.54 \%$ of the variation in SBP and DBP, respectively, after adjustment for age, body mass index, gender, and the top ten genetic principal components (to account for ancestry). A composite score that included the five SNPs along with the 29 ICBP variants [26] explained 0.80 and $1.42 \%$ of the variation in SBP and DBP. This illustrates that GRSs constructed from SNPs identified in EA-only metaanalyses are often associated with the same traits in other ethnicities but to a lesser degree than in EA samples. In addition, ethnicity-specific SNP identification often leads to an increase in predictive capacity for GRSs, underscoring the need for GWAS meta-analyses that include multiple ethnic groups.

A GWAS meta-analysis with over 80,000 Han Chinese (including discovery and replication samples) identified several SNPs that met genome-wide significance for association with SBP, DBP, and/or HTN [14]. A GRS was constructed that included these SNPs as well as SNPs from previous GWAS meta-analyses that were conducted in EA-only or East Asian-only samples. Prior to inclusion in the GRS, SNPs identified in the EA or East Asian meta-analyses were screened to have at least nominally significant associations with BP in the Han Chinese sample. In a subset of $>28,000$ subjects, the GRS was significantly associated with HTN (OR $=1.66$ for the highest versus lowest quintile of GRS) and was also significantly associated with SBP and DBP. This study illustrates a well-powered hybrid approach to GRS SNP selection for use in non-EA ethnic groups.

\section{Intermediate Trait-GRSs Predicting Cardiovascular Outcomes}

In order to gain a better understanding of the relative genetic contribution of specific intermediate pathways or risk factors to cardiovascular traits, GRSs constructed from SNPs associated with intermediate traits (e.g., CAC) may be evaluated for association with cardiovascular outcomes such as CHD, stroke, and CVD. This approach can augment or extend findings from studies using GRSs constructed from trait-specific SNPs. For example, GRSs constructed from BP-associated SNPs (SBP and DBP separately) were associated with incident CHD, IS, and CVD in a large cohort of Finnish subjects (HRs for $\mathrm{CHD}=1.25$ and 1.23; HRs for $\mathrm{IS}=1.25$ and 1.35; HRs for composite $\mathrm{CVD}=1.23$ and 1.26 for $\mathrm{GRS}_{\mathrm{SBP}}$ and $\mathrm{GRS}_{\mathrm{DBP}}$, respectively). This study illustrates that the genetic factors that influence BP also have a significant effect on clinical outcomes [28].

In some cases, constructing GRS based on intermediate traits may be the only option due to the relative lack of studies 
or replicable significant findings from meta-analyses for the trait itself. For example, large-scale meta-analyses of stroke or IS are just beginning to emerge ([29, 30], but the sample sizes are typically smaller for this cardiovascular trait than other CVD outcomes (such as CHD) or for intermediate traits (such as BP or lipids). Several studies have evaluated the relationship between intermediate trait or risk factor-based GRSs and IS. For example, a GRS constructed from SNPs associated with BP was significantly associated with IS in a Swedish sample of 3,677 stroke cases and 2,415 controls $(\mathrm{OR}=1.09$ per SD increase in GRS) [31]. The addition of the GRS demonstrated weak but significant improvement in risk reclassification. A GRS constructed from atrial fibrillation-associated SNPs was also significantly associated with incident IS in Swedish participants (HR=1.23 comparing top and bottom quintiles of GRS) and modestly but significantly improved risk discrimination and reclassification [32]. A GRS constructed from high-density lipoprotein cholesterol (HDL-C) SNPs, however, was not associated with incident IS in European Americans [33].

Recent studies have now gone further to combine SNPs associated with multiple intermediate traits into a composite risk score. For instance, Malik et al. combined SNPs credibly associated with atrial fibrillation, CAD, HTN, and SBP into a single 113-SNP GRS and found that it was significantly associated with IS in both clinic-based case-control and population-based samples $(\mathrm{OR}=1.06$ per GRS SD in casecontrol sample) [34]. Adding the GRS improved prediction of IS beyond a sex-adjusted model in the case-control sample but not in the population-based sample. Another emerging strategy for creating a composite GRS is to combine intermediate trait-associated SNPs with SNPs identified for the trait itself. In a meta-analysis of four population-based EA cohorts, a 324-SNP GRS comprised of SNPs credibly associated with stroke and nine stroke risk factors improved discrimination and risk reclassification for IS beyond a well-validated risk factor prediction model [35॰].

Studies have also evaluated whether GRSs constructed from CHD-associated SNPs only, intermediate traitassociated SNPs only, or the combination of both types of SNPs are predictive of CHD [25, 36]. A case-control study of individuals in the Netherlands found that the best predictor of CHD was a weighted 29-SNP GRS consisting of CHDassociated SNPs only, with an $\mathrm{HR}=1.12$ per risk allele after adjustment for traditional risk factors [36]. Other GRSs that included intermediate trait SNPs were less strongly associated with CHD and were attenuated after risk factor adjustment. A separate study in Swedes, however, found that the CHDspecific GRS and the GRS constructed from CHD-plus intermediate trait-associated SNPs were similarly associated with CHD (HR $=1.5$ for first vs. fourth quartile of GRS) after adjustment for traditional risk factors, and that risk reclassification was modestly but significantly improved beyond traditional risk factors for both GRSs, although the AUC was not [25]. Taken together, these studies suggest that adding SNPs from intermediate traits to a trait-specific GRS may not improve prediction of cardiovascular outcomes.

\section{GRSs Predicting CVD as a Composite Phenotype}

To date, there have been few consortium-based GWASs that use a composite CVD phenotype (including both heart disease and stroke) as the outcome measure, although some are beginning to emerge [37•]. Instead, GWAS meta-analyses have focused on specific CVD endpoints (such as CAD/CHD, MI, or stroke), subclinical CVD phenotypes (such as CAC, IMT, and plaque), and intermediate phenotypes (for a review, see [38]). Following this trend, many GRSs evaluated for their prediction of CVD have been constructed using these traitspecific SNPs from consortium-based GWAS meta-analyses. Studies that specifically evaluate the shared genetic variation between CAD and stroke may lead to a more refined set of SNPs that best predict composite CVD.

In early work on CVD, Paynter et al. constructed an unweighted 12-SNP GRS from published associations with CVD-related endpoints ( $p<10^{-7}$ in meta-analysis) and found a significant relationship with incident CVD in EA women (per-allele $\mathrm{HR}=1.05$ ), although this association was attenuated upon adjustment for traditional risk factors [39]. A second unweighted 101-SNP GRS also included SNPs associated with intermediate traits (cholesterol, BP, diabetes, etc.), but the effect of this GRS on CVD was weaker (per-allele HR= 1.02). Thanassoulis et al. found a significant association between incident CVD and an unweighted 13-SNP GRS constructed from MI/CHD-associated SNPs, with $\mathrm{HR}=1.05$ per allele after adjustment for CVD risk factors and parental history of CVD in the Framingham Heart Study [40]. However, an unweighted 102-SNP GRS that included SNPs associated with intermediate traits was not associated with CVD. These studies show that GRSs with CHD/CAD-associated SNPs are more strongly predictive of composite CVD than more comprehensive GRSs that include intermediate trait-associated SNPs. The same 13-SNP GRS used in Thanassoulis was also found to be significantly associated with prior CVD $(\mathrm{OR}=$ $1.51)$ as well as CVD mortality ( $\mathrm{HR}=1.35)$ in EAs with diabetes, after adjustment for CVD risk factors [41]. In all studies, the GRS failed to improve discrimination, although it did modestly improve risk reclassification of some CVD cases in the latter two studies.

\section{GRSs Associated with Subclinical Measures of Heart Disease}

Since only a very small number of SNPs have been reliably associated with artery calcification and IMT, studies have primarily focused on the evaluation of GRSs constructed from 
$\mathrm{CAD} / \mathrm{MI}$ and/or other risk factor-associated SNPs with subclinical measures of heart disease. In an EA cohort, a GRS created from three SNPs that have been credibly associated with CAC explained $2.4 \%$ of the variation in CAC, and a 45 SNP GRS constructed from CAD-associated SNPs explained an additional $4 \%$ [6]. Another study conducted in EA found that a GRS from the same three CAC-associated SNPs was associated with calcification in multiple vessel beds, but the associations were no longer significant after adjusting for traditional cardiovascular risk factors [42]. A 132-SNP GRS created from SNPs associated with lipids was also associated with vessel bed calcification, though less strongly than the GRS that contained only CAC-associated SNPs. A third study conducted primarily in EAs found a relationship between plaque and GRSs constructed from lipid-associated SNPs but very limited associations between those GRSs and IMT [43]. However, a separate study found that IMT was associated with a GRS that included five fasting glucose-associated SNPs (beta $=0.0048 \mathrm{~mm}$ per GRS SD) [44]. Overall, these studies illustrate that SNPs associated with intermediate traits may be useful for explaining variation in subclinical phenotypes but that more work is needed to identify the SNPs most strongly associated with artery calcification and IMT.

\section{Emerging Uses of GRS}

\section{Prediction in Clinical and High-Risk Populations}

Currently, there is interest in exploring whether GRSs are associated with CVD outcomes and subclinical phenotypes in clinical and other high-risk populations. Accurate prediction of CVD events in high-risk populations, such as those with comorbidities, is imperative because patients are treated according to their risk classification. Initial investigations indicate that GRSs are associated with CVD in populations with comorbidities. For example, as discussed previously, GRSs constructed from CAD/CVDassociated SNPs are associated with CVD, CAC, and CVD mortality in EAs with diabetes, even after adjusting for traditional risk factors [41]. There has also been interest in investigating whether GRSs may be useful for secondary prevention, but most studies have indicated that GRSs are not particularly successful at predicting new CVD events in patients with previous CVD. For example, in a study of 5,742 patients with symptomatic vascular disease, a 30-SNP GRS constructed from CAD-associated SNPs was not able to significantly improve 10-year risk prediction of a composite CVD outcome consisting of MI, stroke, and vascular death [45•]. In a separate study of subjects undergoing heart catheterization, GRSs constructed from CAD/MI-associated SNPs were associated with prevalent, but not incident, MI [46]. More work is needed to assess whether the use of GRSs will translate to clinical utility in terms of risk assessment and ultimately differential treatment in high-risk populations.

\section{GRS-by-Environment Interaction Studies}

Cardiovascular disease is likely to be due, in part, to interaction between genetic and non-genetic components [47], including demographic, dietary, behavioral, environmental, and social factors. Studies of common, chronic diseases have recently begun to utilize GRSs as the genetic variation in gene-by-environment interaction studies, since GRSs cumulatively explain more trait variation than individual SNPs. GRS-by-environment interaction studies are emerging in obesity, type 2 diabetes, and lipids research. For instance, GRSby-age and GRS-by-BMI interactions have been reported for type 2 diabetes [48, 49], and a GRS-by-education interaction was observed for hemoglobin A1c [50]. Several studies have noted GRS-by-diet interactions, including a GRS-by-sugar sweetened beverages interaction for BMI and obesity [51], a GRS-by-macronutrient intake interaction for adiposity traits [52], and a GRS-by-adiposity interaction for triglycerides and HDL-C [53]. This avenue of research is likely to lead to a greater understanding of the etiological factors that underlie the development of complex diseases and thus represents a promising direction for cardiovascular research.

\section{GRSs as Instrumental Variables in Mendelian Randomization Studies}

Mendelian randomization is a method for obtaining an unbiased estimate of the potential causal effect of a risk factor on an outcome of interest using observational data. With this approach, genetic variants are used as an instrumental variable, or a proxy, for the risk factor. GRSs have become a popular choice for instrumental variables because they typically explain more trait variation than single SNPs [10••]. Recent applications of GRSs in Mendelian randomization studies for cardiovascular diseases include the use of a 14-SNP GRS to explore the causal relationship between HDL-C and MI [54] and an 8-SNP GRS to evaluate the relationship between uric acid and multiple cardiometabolic phenotypes [55]. Burgess and Thompson thoroughly review the use of GRSs as instrumental variables in Mendelian randomization studies and provide simulation studies and recommendations for use [10••]. The extension of Mendelian randomization techniques to other data types, such as epigenetic and metabolomic data, may also be a promising area of research for cardiovascular disease and is discussed in [56]. 


\section{Conclusions}

As we take stock of the findings from GWAS meta-analyses conducted over the past decade, the GRS has been one of the most promising ways to aggregate multiple sets of results into a single genetic predictor for cardiovascular disease. More work is needed to identify the genetic factors associated with subclinical phenotypes and cardiovascular outcomes, especially in non-EA populations, so that GRSs can most effectively capture relevant genetic variation. Questions remain about the ultimate clinical utility of the GRS, but further investigation in high-risk populations and new ways to combine GRSs with traditional risk factors may prove to be fruitful.

Acknowledgments The authors would like to thank Belinda Needham for critical review of the manuscript. Funding was provided by the National Heart, Lung and Blood Institute (R01 HL119443), the National Institute on Aging (R03 AG046389), and the National Institute on Minority Health and Health Disparities (P60 MD002249).

\section{Compliance with Ethics Guidelines}

Conflict of Interest JA Smith, EB Ware, P Middha, L Beacher, and SLR Kardia all declare no conflicts of interest.

Human and Animal Rights and Informed Consent All studies by JA Smith and SLR Kardia involving animal and/or human subjects were performed after approval by the appropriate institutional review boards. When required, written informed consent was obtained from all participants.

Open Access This article is distributed under the terms of the Creative Commons Attribution 4.0 International License (http:// creativecommons.org/licenses/by/4.0/), which permits unrestricted use, distribution, and reproduction in any medium, provided you give appropriate credit to the original author(s) and the source, provide a link to the Creative Commons license, and indicate if changes were made.

\section{References}

Papers of particular interest, published recently, have been highlighted as:

- Of importance

•- Of major importance

1. Wray NR, Goddard ME, Visscher PM. Prediction of individual genetic risk to disease from genome-wide association studies. Genome Res. 2007;17(10):1520-8.

2. Humphries SE, Yiannakouris N, Talmud PJ. Cardiovascular disease risk prediction using genetic information (gene scores): is it really informative? Curr Opin Lipidol. 2008;19(2):128-32.

3. Mozaffarian D, Benjamin EJ, Go AS, et al. Heart disease and stroke statistics-2015 update: a report from the American Heart Association. Circulation. 2015;131(4):e29-322.

4. Morrison AC, Bare LA, Chambless LE, et al. Prediction of coronary heart disease risk using a genetic risk score: the Atherosclerosis Risk in Communities Study. Am J Epidemiol. 2007;166(1):28-35.
5. Brautbar A, Pompeii LA, Dehghan A, et al. A genetic risk score based on direct associations with coronary heart disease improves coronary heart disease risk prediction in the Atherosclerosis Risk in Communities (ARIC), but not in the Rotterdam and Framingham Offspring, Studies. Atherosclerosis. 2012;223(2):421-6.

6. van Setten J, Isgum I, Pechlivanis S, et al. Serum lipid levels, body mass index, and their role in coronary artery calcification: a polygenic analysis. Circ Cardiovasc: Genet; 2015. This study shows that adding thousands of SNPs marginally associated with CHD to a genetic risk score can increase the percent of variation explained in a sub-clinical phenotype.

7. Vassy JL, Hivert MF, Porneala B, et al. Polygenic type 2 diabetes prediction at the limit of common variant detection. Diabetes. 2014;63(6):2172-82.

8.• Belsky DW, Moffitt TE, Sugden K, et al. Development and evaluation of a genetic risk score for obesity. Biodemography Soc Biol. 2013;59(1):85-100. This study provides a new method for generating genetic risk scores for complex traits through the use of multiple sources of information such as publicly available GWAS databases and web-based GWAS analysis tools.

9. Hivert MF, Vassy JL, Meigs JB. Susceptibility to type 2 diabetes mellitus-from genes to prevention. Nat Rev Endocrinol. 2014;10(4):198-205.

10.• Burgess S, Thompson SG. Use of allele scores as instrumental variables for Mendelian randomization. Int J Epidemiol. 2013;42(4):1134-44. This article reviews the use of genetic risk scores as instrumental variables in Mendelian randomization studies, which can be used to estimate the causal effect of a risk factor on an outcome of interest.

11. Li YR, Keating BJ. Trans-ethnic genome-wide association studies: advantages and challenges of mapping in diverse populations. Genome Med. 2014;6(10):91-014-0091-5. eCollection 2014.

12. CARDIoGRAMplusC4D Consortium, Deloukas P, Kanoni S, et al. Large-scale association analysis identifies new risk loci for coronary artery disease. Nat Genet. 2013;45(1):25-33.

13. Franceschini N, Fox E, Zhang Z, et al. Genome-wide association analysis of blood-pressure traits in African-ancestry individuals reveals common associated genes in African and non-African populations. Am J Hum Genet. 2013;93(3):545-54.

14. Lu X, Wang L, Lin X, et al. Genome-wide association study in Chinese identifies novel loci for blood pressure and hypertension. Hum Mol Genet. 2015;24(3):865-74.

15. Gui L, Wu F, Han X, et al. A multilocus genetic risk score predicts coronary heart disease risk in a Chinese Han population. Atherosclerosis. 2014;237(2):480-5.

16. Cook NR, Ridker PM. Advances in measuring the effect of individual predictors of cardiovascular risk: the role of reclassification measures. Ann Intern Med. 2009;150(11):795-802.

17. Hanley JA, McNeil BJ. The meaning and use of the area under a receiver operating characteristic (ROC) curve. Radiology. 1982;143(1):29-36.

18. DeLong ER, DeLong DM, Clarke-Pearson DL. Comparing the areas under two or more correlated receiver operating characteristic curves: a nonparametric approach. Biometrics. 1988;44(3):837-45.

19. Cook NR. Use and misuse of the receiver operating characteristic curve in risk prediction. Circulation. 2007;115(7):928-35.

20. Pencina MJ, D'Agostino RBS, D'Agostino Jr RB, Vasan RS. Evaluating the added predictive ability of a new marker: from area under the ROC curve to reclassification and beyond. Stat Med. 2008;27(2):157-72.

21. Pencina MJ, D'Agostino RBS, Steyerberg EW. Extensions of net reclassification improvement calculations to measure usefulness of new biomarkers. Stat Med. 2011;30(1):11-21.

22.• Kerr KF, Wang Z, Janes H, McClelland RL, Psaty BM, Pepe MS. Net reclassification indices for evaluating risk prediction instruments: a critical review. Epidemiology. 2014;25(1):114-21. This 
critical review of net reclassification indices (NRI) discusses appropriate interpretations of NRIs, outlines their advantages and disadvantages, and makes recommendations for their use in measuring prediction for new biomarkers.

23. Cook NR. Comments on 'evaluating the added predictive ability of a new marker: from area under the ROC curve to reclassification and beyond' by M. J. Pencina et al. Stat Med. 2008;27(2):191-5.

24. Tikkanen E, Havulinna AS, Palotie A, Salomaa V, Ripatti S. Genetic risk prediction and a 2-stage risk screening strategy for coronary heart disease. Arterioscler Thromb Vasc Biol. 2013;33(9):2261-6.

25. Ganna A, Magnusson PK, Pedersen NL, et al. Multilocus genetic risk scores for coronary heart disease prediction. Arterioscler Thromb Vasc Biol. 2013;33(9):2267-72.

26. International Consortium for Blood Pressure Genome-Wide Association Studies, Ehret GB, Munroe PB, et al. Genetic variants in novel pathways influence blood pressure and cardiovascular disease risk. Nature. 2011;478(7367):103-9.

27. Fava C, Sjogren M, Montagnana M, et al. Prediction of blood pressure changes over time and incidence of hypertension by a genetic risk score in Swedes. Hypertension. 2013;61(2):319-26.

28. Havulinna AS, Kettunen J, Ukkola O, et al. A blood pressure genetic risk score is a significant predictor of incident cardiovascular events in 32,669 individuals. Hypertension. 2013;61(5):987-94.

29. International Stroke Genetics Consortium (ISGC), Wellcome Trust Case Control Consortium 2 (WTCCC2), Bellenguez C, et al. Genome-wide association study identifies a variant in HDAC9 associated with large vessel ischemic stroke. Nat Genet. 2012;44(3): 328-33.

30. Traylor M, Farrall M, Holliday EG, et al. Genetic risk factors for ischaemic stroke and its subtypes (the METASTROKE collaboration): a meta-analysis of genome-wide association studies. Lancet Neurol. 2012;11(11):951-62.

31. Fava C, Sjogren M, Olsson S, et al. A genetic risk score for hypertension associates with the risk of ischemic stroke in a Swedish case-control study. Eur J Hum Genet. 2014. doi:10.1038/ejhg. 2014.212.

32. Tada H, Shiffman D, Smith JG, et al. Twelve-single nucleotide polymorphism genetic risk score identifies individuals at increased risk for future atrial fibrillation and stroke. Stroke. 2014;45(10): 2856-62.

33. Pikula A, Beiser AS, Wang J, et al. Lipid and lipoprotein measurements and the risk of ischemic vascular events: Framingham Study. Neurology. 2015;84(5):472-9.

34. Malik R, Bevan S, Nalls MA, et al. Multilocus genetic risk score associates with ischemic stroke in case-control and prospective cohort studies. Stroke. 2014;45(2):394-402.

35. Ibrahim-Verbaas CA, Fornage M, Bis JC, et al. Predicting stroke through genetic risk functions: the CHARGE Risk Score Project. Stroke. 2014;45(2):403-12. This study illustrates the use of genetic risk scores composed of SNPs associated with intermediate traits to predict cardiovascular events.

36. Vaarhorst AA, Lu Y, Heijmans BT, et al. Literature-based genetic risk scores for coronary heart disease: the Cardiovascular Registry Maastricht (CAREMA) prospective cohort study. Circ Cardiovasc Genet. 2012;5(2):202-9.

37. Dichgans M, Malik R, Konig IR, et al. Shared genetic susceptibility to ischemic stroke and coronary artery disease: a genome-wide analysis of common variants. Stroke. 2014;45(1):24-36. This study evaluates the shared genetic contribution to ischemic stroke (IS) and coronary artery disease (CAD), including a meta-analysis on a composite cardiovascular phenotype (combining both CAD and IS).

38. Gustav Smith J, Newton-Cheh C. Genome-wide association studies of late-onset cardiovascular disease. J Mol Cell Cardiol. 2015. This article provides a review of the findings from GWASs conducted for late-onset cardiovascular disease, including coronary artery disease and ischemic stroke.

39. Paynter NP, Chasman DI, Pare G, et al. Association between a literature-based genetic risk score and cardiovascular events in women. JAMA. 2010;303(7):631-7.

40. Thanassoulis G, Peloso GM, Pencina MJ, et al. A genetic risk score is associated with incident cardiovascular disease and coronary artery calcium: the Framingham Heart Study. Circ Cardiovasc Genet. 2012;5(1):113-21

41. Cox AJ, Hsu FC, Ng MC, et al. Genetic risk score associations with cardiovascular disease and mortality in the Diabetes Heart Study. Diabetes Care. 2014;37(4):1157-64.

42. Bos D, Ikram MA, Isaacs A, et al. Genetic loci for coronary calcification and serum lipids relate to aortic and carotid calcification. Circ Cardiovasc Genet. 2013;6(1):47-53.

43. Isaacs $\mathrm{A}$, Willems $\mathrm{SM}, \mathrm{Bos} \mathrm{D}$, et al. Risk scores of common genetic variants for lipid levels influence atherosclerosis and incident coronary heart disease. Arterioscler Thromb Vasc Biol. 2013;33(9): 2233-9.

44. Rasmussen-Torvik LJ, Li M, Kao WH, et al. Association of a fasting glucose genetic risk score with subclinical atherosclerosis: The Atherosclerosis Risk in Communities (ARIC) study. Diabetes. 2011;60(1):331-5.

45. Weijmans M, de Bakker PI, van der Graaf Y, et al. Incremental value of a genetic risk score for the prediction of new vascular events in patients with clinically manifest vascular disease. Atherosclerosis. 2015;239(2):451-8. This study is an example of using genetic risk scores to predict cardiovascular events in a clinical population.

46. Patel RS, Sun YV, Hartiala J, et al. Association of a genetic risk score with prevalent and incident myocardial infarction in subjects undergoing coronary angiography. Circ Cardiovasc Genet. 2012;5(4):441-9.

47. Flowers E, Froelicher ES, Aouizerat BE. Gene-environment interactions in cardiovascular disease. Eur J Cardiovasc Nurs. 2012;11(4):472-8.

48. Langenberg C, Sharp SJ, Franks PW, et al. Gene-lifestyle interaction and type 2 diabetes: the EPIC interact case-cohort study. PLoS Med. 2014;11(5):e1001647.

49. Cornelis MC, Qi L, Zhang C, et al. Joint effects of common genetic variants on the risk for type 2 diabetes in U.S. men and women of European ancestry. Ann Intern Med. 2009;150(8):541-50.

50. Liu SY, Walter S, Marden J, et al. Genetic vulnerability to diabetes and obesity: does education offset the risk? Soc Sci Med. 2015;127: 1501-58.

51. Qi Q, Chu AY, Kang JH, et al. Sugar-sweetened beverages and genetic risk of obesity. N Engl J Med. 2012;367(15):1387-96.

52. Goni L, Cuervo M, Milagro FI, Martinez JA. A genetic risk tool for obesity predisposition assessment and personalized nutrition implementation based on macronutrient intake. Genes Nutr. 2015;10(1):445.

53. Cole CB, Nikpay M, Lau P, et al. Adiposity significantly modifies genetic risk for dyslipidemia. J Lipid Res. 2014;55(11):2416-22.

54. Voight BF, Peloso GM, Orho-Melander M, et al. Plasma HDL cholesterol and risk of myocardial infarction: a mendelian randomisation study. Lancet. 2012;380(9841):572-80.

55. Kleber ME, Delgado G, Grammer TB, et al. Uric acid and cardiovascular events: a mendelian randomization study. J Am Soc Nephrol. 2015

56. Brion MA, Benyamin B, Visscher PM, Davey SG. Beyond the single SNP: emerging developments in Mendelian randomization in the "Omics" era. Curr Epidemiol Rep. 2014;1:228-36.

57. Hindorff J, MacArthur J, Morales J, et al. A catalog of published genome-wide association studies. www.genome.gov/gwastudies.

58. O'Donnell CJ, Kavousi M, Smith AV, et al. Genome-wide association study for coronary artery calcification with follow-up in myocardial infarction. Circulation. 2011;124(25):2855-64. 
59. Teslovich TM, Musunuru K, Smith AV, et al. Biological, clinical and population relevance of 95 loci for blood lipids. Nature. 2010;466(7307):707-13.

60. Global Lipids Genetics Consortium, Willer CJ, Schmidt EM, et al. Discovery and refinement of loci associated with lipid levels. Nat Genet. 2013;45(11):1274-83.
61. Speliotes EK, Willer CJ, Berndt SI, et al. Association analyses of 249,796 individuals reveal 18 new loci associated with body mass index. Nat Genet. 2010;42(11):937-48.

62. Morris AP, Voight BF, Teslovich TM, et al. Large-scale association analysis provides insights into the genetic architecture and pathophysiology of type 2 diabetes. Nat Genet. 2012;44(9):981-90. 